\title{
Comparison between the growth of Brazilian children and adolescents and the reference growth charts: data from a Brazilian project
}

\author{
Comparação do crescimento de crianças e adolescentes brasileiros \\ Diego Augusto Santos Silva', Andreia Pelegrini², \\ Edio Luiz Petroski ${ }^{3}$, Adroaldo Cesar Araujo Gaya ${ }^{4}$
} com curvas de referência para crescimento físico: dados do Projeto Esporte Brasil

\begin{abstract}
Resumo
Objetivo: Comparar crianças e adolescentes brasileiros com curvas de referência para crescimento físico.

Métodos: Estudo transversal, de base escolar, conduzido em 41.654 estudantes ( 23.328 do sexo masculino e 18.326 do sexo feminino) de 7 a 17 anos. Foram mensuradas variáveis antropométricas (estatura, peso corporal) e calculado o índice de massa corporal (IMC). A estatura, percentil 50, e o IMC, percentil 85, foram comparados com tabelas normativas da Organização Mundial de Saúde (OMS), e o peso corporal, percentil 50, com as curvas do Center for Disease Control and Prevention (CDC).

Resultados: Ao comparar os valores de estatura e peso corporal de ambos os sexos com os valores de referência da OMS e do CDC foi identificado que os jovens brasileiros atingiram e ultrapassaram os pontos de referência na maioria das idades. Os IMC dos escolares brasileiros foram inferiores aos valores de sobrepeso da OMS para todas as idades.

Conclusão: A magnitude de crescimento em peso corporal, estatura e IMC de crianças e adolescentes da zona urbana do Brasil está cada vez mais semelhante à reportada em países desenvolvidos. Sugere-se que estudos prospectivos no Brasil sejam realizados e comparados com curvas internacionais de crescimento físico a fim de poder realizar inferências mais precisas.
\end{abstract}

J Pediatr (Rio J). 2010;86(2):115-120: Crescimento, antropometria, saúde da criança, saúde do adolescente, estudos transversais.

\begin{abstract}
Objective: To compare the growth of Brazilian children and adolescents with reference growth charts.

Methods: School-based cross-sectional study involving 41,654 students (23,328 boys and 18,326 girls) aged 7 to 17 years. Physical growth variables (body weight, height) and body mass index (BMI) were measured. Height, 50th percentile, and BMI, 85th percentile, were compared with the World Health Organization (WHO) reference values, and weight, 50th percentile, was compared with the Centers for Disease Control and Prevention (CDC) growth charts.

Results: Mean height and body weight of Brazilian schoolchildren were generally higher than the WHO and CDC reference values for both genders at most ages. BMI values of Brazilian schoolchildren were significantly lower than the WHO obesity values at all ages.

Conclusion: The growth in body weight, height, and BMI of children and adolescents in the urban area of Brazil is increasingly similar to those reported in developed countries. Further prospective studies should be conducted in Brazil comparing their results with the international growth charts to enable more accurate inferences.
\end{abstract}

JPediatr (Rio J). 2010;86(2):115-120: Growth, anthropometry, child health, adolescent health, cross-sectional studies.

1. Professor, Educação Física, Programa de Pós-Graduação em Educação Física, Núcleo de Pesquisa em Cineantropometria e Desempenho Humano (NuCIDH), Universidade Federal de Santa Catarina (UFSC), Florianópolis, SC. Bolsista CAPES.

2. Mestre, Educação Física, Programa de Pós-Graduação em Educação Física, NuCIDH, UFSC, Florianópolis, SC. Bolsista CAPES.

3. Doutor, Educação Física, Programa de Pós-Graduação em Educação Física, NuCIDH, UFSC, Florianópolis, SC.

4. Doutor, Educação Física, Universidade Federal do Rio Grande do Sul (UFRGS), Porto Alegre, RS. Coordenador, Projeto Esporte Brasil.

Apoio financeiro: Ministério do Esporte/Projeto Esporte Brasil.

Não foram declarados conflitos de interesse associados à publicação deste artigo.

Como citar este artigo: Silva DA, Pelegrini A, Petroski EL, Gaya AC. Comparison between the growth of Brazilian children and adolescents and the reference growth charts: data from a Brazilian project. J Pediatr (Rio J). 2010;86(2):115-120.

Artigo submetido em 20.08.09, aceito em 04.12.09.

doi:10.2223/JPED.1975 


\section{Introdução}

A monitoração do crescimento físico ocorre tradicionalmente por meio de medidas antropométricas de peso corporal e estatura, as quais apresentam aceitação internacional para esse fim ${ }^{1}$. Com estas medidas, agências de saúde, como o Center for Disease Control and Prevention (CDC) ${ }^{2}$ dos EUA, desenvolveram curvas normativas de crescimento físico utilizadas por muito tempo como referência em diversos países. Outros países (Itália ${ }^{3}$, Arábia Saudita ${ }^{4}$, Irã ${ }^{5}$, Turquia $^{6}$, Índia ${ }^{7}$ e Grécia $^{8}$ ) criaram suas próprias curvas de crescimento, mas, mesmo assim, realizam comparações com os pontos de referência do CDC.

Algumas críticas às curvas de crescimento do CDC foram realizadas, como, por exemplo, as relativas à amostra oriunda exclusivamente dos EUA e à subestimação do crescimento físico de crianças saudáveis ${ }^{9}$. Nesse sentido, a Organização Mundial de Saúde (OMS) desenvolveu um conjunto de curvas de crescimento ${ }^{10}$ criadas a partir de um estudo em quatro continentes que levou em consideração a diversidade étnica, condições de vida semelhantes das crianças avaliadas nos diferentes países, mesmo delineamento amostral e métodos epidemiológicos e estatísticos atualizados para garantir o controle e qualidade das curvas.

No Brasil, foram conduzidas iniciativas para a criação de uma curva de crescimento11,12. Uma delas foi realizada no estado de São Paulo ${ }^{11}$ e a outra, pelo Instituto Nacional de Nutrição ${ }^{12}$. A primeira, publicada em 1982, e a segunda, em 1990, necessitam de uma revisão, tendo em vista o efeito da tendência secular no crescimento ${ }^{13}$. Além destas iniciativas, estudos isolados em diferentes regiões e cidades foram desenvolvidos ${ }^{14-16}$

Por algum tempo, as curvas adotadas no Brasil como referência eram as do $\mathrm{CDC}^{2}$; entretanto, a partir da publicação dos valores oriundos da $\mathrm{OMS}^{10}$, o Ministério da Saúde recomendou a utilização dessas curvas no país. Poucos estudos comparativos com amostras brasileiras utilizando as recentes curvas de crescimento da OMS foram desenvolvidos ${ }^{16}$. Algumas investigações realizaram comparações com os dados do $\mathrm{CDC}^{14,15}$. No entanto, tais levantamentos foram realizados em populações isoladas, devido à dificuldade de recrutar uma amostra das diferentes regiões do Brasil.

Assim, o presente estudo objetiva comparar crianças e adolescentes brasileiros com valores de referência para crescimento físico do CDC e da OMS.

\section{Métodos}

Para este estudo transversal, de base escolar, os dados foram extraídos do Projeto Esporte Brasil (PROESP-BR), um observatório permanente dos indicadores de crescimento, desenvolvimento e estado nutricional de crianças e adolescentes brasileiros de 7 a 17 anos, que tem como principal objetivo delinear o perfil somatomotor, hábitos de vida e fatores de aptidão motora em crianças e adolescentes, tendo em vista agregar indicadores para subsidiar políticas de Educação Física e Esportes para crianças e jovens no Brasil.
O PROESP-BR faz parte de um conjunto de projetos realizados pela rede dos Centros de Excelência Esportiva (CENESP), ligada ao Departamento de Excelência Esportiva e Promoção de Eventos da Secretaria Nacional de Esporte de Alto Rendimento do Ministério do Esporte. Informações mais detalhadas sobre a concepção e aspectos metodológicos do PROESP-BR foram publicadas previamente ${ }^{17}$. O projeto foi aprovado pelo Comitê de Ética em Pesquisas com Seres Humanos da Universidade Federal de Santa Catarina (UFSC), Florianópolis (SC) (Parecer Substanciado - 218/08).

A população do presente estudo consistiu de estudantes de 7 a 17 anos matriculados na rede pública e privada de ensino das cinco regiões brasileiras (Norte, Nordeste, Centro-Oeste, Sudeste e Sul). A seleção da amostra foi realizada de forma não probabilística e intencional, de modo que o PROESP-BR é divulgado nas escolas e os professores de Educação Física têm a opção de aderir, avaliando os alunos e encaminhando os dados à coordenação do projeto.

No ano de 2004/2005, integraram o banco de dados do PROESP-BR informações sobre estudantes de 23 estados do Brasil e do Distrito Federal. Foram coletados dados em três estados da Região Centro-Oeste [Goiás ( $n=1.835)$, Mato Grosso ( $n=1.438$ ) e Mato Grosso do Sul ( $n=1.023)$ ] e no Distrito Federal ( $n=2.473$ ), em oito estados da Região Nordeste [Alagoas $(n=401)$, Bahia $(n=984)$, Maranhão $(n=655)$, Paraíba $(n=226)$, Pernambuco $(n=1.300)$, Piauí ( $n=1.034)$, Rio Grande do Norte $(n=905)$ e Sergipe $(n=280)$ ], cinco estados da Região Norte [Amazonas ( $n=492)$, Amapá $(n=1.094)$, Pará $(n=1.141)$, Rondônia ( $n=353$ ) e Tocantins $(n=791)$ ], quatro estados da Região Sudeste [Espírito Santo $(n=371)$, Minas Gerais $(n=2.526)$, Rio de Janeiro ( $n=4.361)$ e São Paulo $(n=7.507)$ ] e em três estados da Região Sul [Paraná ( $n=1.540)$, Santa Catarina ( $n=91)$ e Rio Grande do Sul $(n=8.833)$. Dos estudantes avaliados, $97,1 \%$ estudavam em escolas localizadas nas zonas urbanas das cidades.

Os dados antropométricos (estatura e peso corporal) foram obtidos pelos professores de Educação Física de cada escola que aderiu ao PROESP-BR. Todos os professores passaram por treinamento e tiveram acesso às instruções de aplicação de testes e medidas por meio de um site na Internet que incluía um vídeo para padronização e melhor apresentação visual das técnicas de medidas ${ }^{17}$, preparado por membros da Escola de Educação Física da Universidade Federal do Rio Grande do Sul (UFRGS), Porto Alegre (RS).

O peso corporal foi determinado por meio de uma balança antropométrica digital graduada de 0 a $150 \mathrm{~kg}$, com resolução de $0,05 \mathrm{~kg}$, e a estatura, por meio de um estadiômetro portátil fixado à parede, graduado de 0 a $200 \mathrm{~cm}$, com escala de precisão de $0,2 \mathrm{~cm}^{17}$. O índice de massa corporal (IMC) foi calculado por meio do quociente da massa corporal em $\mathrm{kg}$ pela estatura em $\mathrm{m}^{2}$.

As médias das variáveis de crescimento físico do presente estudo foram comparadas com os valores de referência da OMS para estatura e IMC ${ }^{10}$ e com os do CDC para o peso corporal $^{2}$. Foi adotada essa divisão, porque a OMS não apresenta dados de peso corporal para adolescentes e, nesse sentido, as curvas do CDC são as referências mais recentes 
para essa variável. Foi considerado como valor de referência para o peso corporal e a estatura o percentil 50 (P-50) das curvas, como preconizado em outros estudos ${ }^{3-4,15}$. Já para o IMC, os valores da OMS considerados como referência foram os do percentil 85 (P-85), pois jovens com IMC acima desses valores são classificados como com excesso de peso ${ }^{10}$.

Para o tratamento dos dados referentes à estatura, peso corporal e IMC foram utilizados, inicialmente, a estatística descritiva (média e desvio padrão) para cada sexo e idade. A análise de variância two way foi empregada para verificar diferenças entre os sexos e as idades. O teste de comparação múltipla de Bonferroni foi adotado para verificar diferenças entre os intervalos etários. O teste $t$ de Student para uma amostra foi empregado na comparação dos valores médios de cada variável de crescimento com os valores de referência do CDC e da OMS. Em todas as análises adotou-se um nível de significância de 5\%.

\section{Resultados}

A amostra do presente estudo foi formada por 41.654 escolares de 7 a 17 anos, com uma média de 12,24士1,98 anos de idade, sendo $44 \%$ do sexo feminino e $56 \%$ do masculino. Em relação à distribuição por sexo, a amostra feminina foi de $15,4,11,0,11,8,33,8$ e $28 \%$, e a masculina, de $17,0,12,2$, $11,2,36,7$ e 22,9\% para as Regiões Centro-Oeste, Nordeste, Norte, Sudeste e Sul, respectivamente.

$\mathrm{Na}$ Tabela 1, são apresentados os valores de média e desvio padrão da estatura, peso corporal e IMC de crianças e adolescentes brasileiros. Ao comparar os sexos em relação à estatura, observaram-se valores estatisticamente superiores para o masculino nas idades de 7, 13, 14, 15, 16 e 17 anos, e, para o feminino, nas idades de 10, 11 e 12 anos. Para o peso corporal, dos 10 aos 12 anos, o sexo feminino apresentou valores superiores aos do masculino, sendo que dos 14 anos em diante isso se inverteu. O IMC diferiu nas idades de $10,12,13,14,15$ e 16 anos, nas quais o sexo feminino apresentou valores superiores aos do masculino.

A Figura 1 apresenta, respectivamente, a comparação da estatura, peso corporal e IMC das crianças e adolescentes do presente estudo com os valores de referência analisados. Os jovens brasileiros apresentaram, na maioria das idades, valores mais elevados que os pontos de referência da OMS para estatura e que os do CDC para o peso corporal $(p \leq 0,05)$. O IMC diferiu em todas as idades e em ambos os sexos, sendo os valores de referência da OMS superiores aos do presente estudo ( $p \leq 0,05)$.

\section{Discussão}

O presente estudo de base escolar é o primeiro do Brasil a fazer uso de uma amostra formada por escolares das cinco regiões e teve como objetivo comparar estudantes brasileiros com valores internacionais de referência para crescimento físico ${ }^{2,10}$. Acredita-se que o fato de a amostra envolver crianças e adolescentes que frequentam a escola não diminuiu a capacidade de análise de jovens brasileiros, podendo servir como bom parâmetro comparativo para investigações futuras devido à amostra ser oriunda das cinco regiões.

Este estudo identificou que a partir dos 10 anos de idade, a estatura começa a diferir entre os sexos. Tais diferenças estão relacionadas ao início da adolescência, acompanhado pelo período do estirão de crescimento que ocorre mais cedo no sexo feminino ${ }^{2,13}$. Malina et al. ${ }^{13}$ realizaram um levanta-

Tabela 1 - Valores descritivos para a estatura, peso corporal e IMC de crianças e adolescentes brasileiros - Projeto Esporte Brasil (Brasil, 2004-2005)

\begin{tabular}{|c|c|c|c|c|c|c|}
\hline \multirow[b]{2}{*}{ Idade (anos) } & \multicolumn{2}{|c|}{ Estatura $(\mathrm{cm})$} & \multicolumn{2}{|c|}{ Peso corporal $(\mathbf{k g})$} & \multicolumn{2}{|c|}{$\operatorname{IMC}\left(\mathrm{kg} / \mathrm{m}^{2}\right)$} \\
\hline & Feminino & Masculino & Feminino & Masculino & Feminino & Masculino \\
\hline 7 & $125,6 \pm 6,9$ & $127,7 \pm 6,9 *$ & $27,0 \pm 5,6$ & $27,5 \pm 5,5$ & $17,0 \pm 2,9$ & $16,8 \pm 2,4$ \\
\hline 8 & $131,0 \pm 8,2$ & $131,6 \pm 7,5$ & $30,1 \pm 7,3$ & $30,0 \pm 7,4$ & $17,4 \pm 3,1$ & $17,2 \pm 3,4$ \\
\hline 9 & $137,7 \pm 6,8$ & $137,4 \pm 7,7$ & $32,0 \pm 7,1$ & $33,4 \pm 8,1^{*}$ & $17,0 \pm 3,1$ & $17,6 \pm 3,4 *$ \\
\hline 10 & $142,4 \pm 7,5^{*}$ & $141,3 \pm 7,3$ & $35,4 \pm 8,1^{*}$ & $34,3 \pm 8,0$ & $17,4 \pm 3,1^{*}$ & $17,1 \pm 3,2$ \\
\hline 11 & $148,8 \pm 7,8^{*}$ & $146,1 \pm 7,7$ & $40,0 \pm 9,0 *$ & $38,9 \pm 9,3$ & $18,0 \pm 3,2$ & $17,9 \pm 3,1$ \\
\hline 12 & $154,3 \pm 7,3 *$ & $152,1 \pm 8,8$ & $45,0 \pm 9,4 *$ & $42,5 \pm 10,4$ & $18,9 \pm 3,3^{*}$ & $18,1 \pm 3,2$ \\
\hline 13 & $157,7 \pm 6,8$ & $158,9 \pm 9,4^{*}$ & $48,2 \pm 9,0$ & $47,8 \pm 11,4$ & $19,4 \pm 3,2 *$ & $18,8 \pm 3,2$ \\
\hline 14 & $160,3 \pm 6,6$ & $165,6 \pm 9,0 *$ & $51,2 \pm 8,2$ & $53,6 \pm 11,5^{*}$ & $20,0 \pm 3,0 *$ & $19,4 \pm 3,1$ \\
\hline 15 & $160,7 \pm 6,7$ & $169,7 \pm 8,3^{*}$ & $53,1 \pm 8,6$ & $57,7 \pm 11,6^{*}$ & $20,6 \pm 3,1 *$ & $19,9 \pm 3,1$ \\
\hline 16 & $163,6 \pm 9,6$ & $173,4 \pm 8,1^{*}$ & $57,7 \pm 11,4$ & $63,1 \pm 10,7 *$ & $21,4 \pm 3,1^{*}$ & $20,9 \pm 2,9$ \\
\hline 17 & $162,5 \pm 6,5$ & $174,2 \pm 7,0^{*}$ & $57,1 \pm 9,1$ & $66,1 \pm 11,2^{*}$ & $21,6 \pm 3,1$ & $21,7 \pm 3,2$ \\
\hline
\end{tabular}

IMC = índice de massa corporal.

${ }^{*} p<0,05$, diferença entre os sexos na mesma idade. 
mento das pesquisas sobre crescimento físico em crianças e adolescentes dos países da América do Norte e da Europa e evidenciaram que o estirão do crescimento ocorre 2 anos
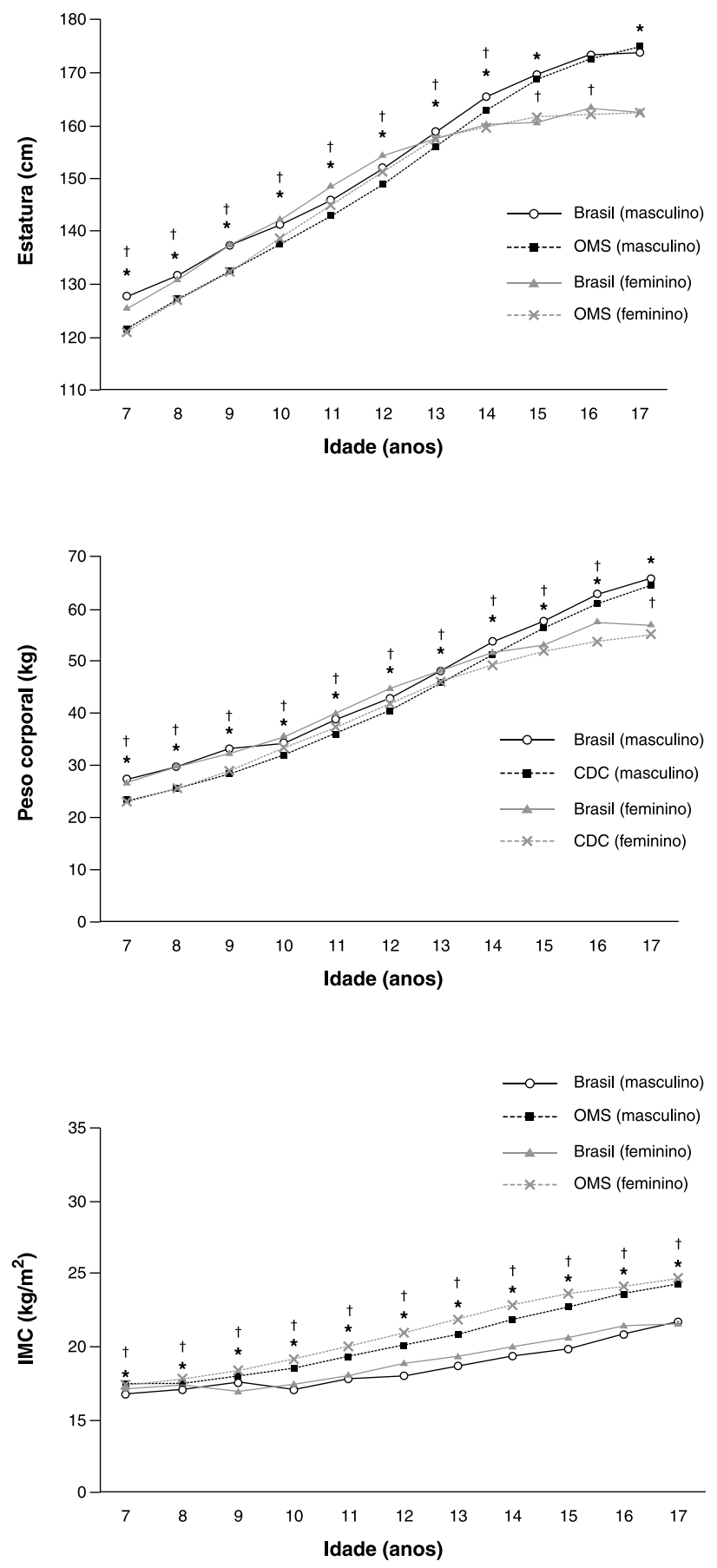

$\mathrm{CDC}=$ Centers for Disease Control and Prevention; IMC = índice de massa corporal; OMS = Organização Mundial da Saúde.

* $p \leq 0,05=$ comparados os valores do sexo masculino do presente estudo com as curvas de referência.

† $p \leq 0,05=$ comparados os valores do sexo feminino do presente estudo com as curvas de referência.

Figura 1 - Comparação da estatura, peso corporal e IMC dos escolares brasileiros com as curvas de referência da OMS e CDC mais tarde no sexo masculino em comparação ao feminino e que esse fato torna os adolescentes mais altos e pesados, quando comparados às adolescentes.

Dados do $\mathrm{CDC}^{2}$, obtidos com a população estadunidense, identificaram que o sexo feminino apresenta valores de estatura superiores aos do masculino por volta dos 11-12 anos de idade, e que 2 anos depois isso se inverte.

Ao comparar o peso corporal entre os sexos foi verificado que dos 10 aos 12 anos os escolares do sexo feminino eram mais pesados que os do masculino, invertendo-se o processo a partir dos 14 anos. Essas diferenças são justificadas por Riggs et al. ${ }^{18}$ e Wells ${ }^{19}$, estando relacionadas às influências hormonais, que afetam mais cedo o sexo feminino do que o masculino, pois o estrogênio (hormônio sexual feminino) causa um alargamento da pelve, desenvolvimento das mamas e aumento da deposição de gordura, principalmente nas coxas e na região do quadril, resultando no aumento do peso corporal. Já a testosterona (hormônio sexual masculino) começa a atuar intensamente no sexo masculino em idade mais tardia do que o estrogênio no sexo feminino e provoca o aumento da formação óssea e da síntese proteica, aumentando a estatura e a massa muscular.

O levantamento desenvolvido pela $\mathrm{OMS}^{10}$ em jovens de nível socioeconômico elevado do Brasil, Gana, Índia, Noruega, Omã e EUA encontrou resultados semelhantes aos do presente estudo ao comparar o peso corporal em relação aos sexos. Na Itália, uma pesquisa que traçou curvas de crescimento físico de crianças e adolescentes ${ }^{3}$ identificou que os valores de peso corporal no sexo feminino eram maiores que os do masculino a partir dos 11 anos de idade e que a partir dos 13 anos o processo se invertia. Deste modo, sugere-se que a magnitude do crescimento em peso corporal de crianças e adolescentes da zona urbana do Brasil está cada vez mais semelhante à reportada em países desenvolvidos, o que não era verificado 2 ou 3 décadas atrás ${ }^{11,12}$.

Ao comparar os valores de estatura e peso corporal de ambos os sexos com os valores de referência da OMS e do CDC, foi identificado que os jovens brasileiros atingiram e ultrapassaram os pontos de referência na maioria das idades. Estudos epidemiológicos conduzidos na Itália ${ }^{3}$, Irã ${ }^{5}$ e Índia7 também verificaram que na maior parte das idades os valores de referência eram inferiores. Em contrapartida, estudos conduzidos na Arábia Saudita ${ }^{4}$ e na Turquia 6 os resultados foram contrários. Em pesquisa conduzida com crianças indígenas ${ }^{16}$ Suruí, Xavánte e Wari, na Amazônia Brasileira, foi constatado que essa população está aquém da estatura para a idade, se comparada com as curvas de crescimento físico da OMS.

Esses achados evidenciam que, além dos fatores genéticos inerentes de cada etnia, as diferentes condições econômicas do país e da população investigada influenciam no crescimento físico de crianças e adolescentes, pois refletem diretamente nos fatores nutricionais, ambientais e maturacionais ligados ao crescimento ${ }^{1,13}$.

Em relação ao IMC, a partir dos 11 e até os 16 anos foram percebidos aumentos significativos para ambos os sexos; entretanto, esses valores são mais elevados no sexo 
feminino. Esse fato deve-se, inicialmente, às alterações provocadas pela puberdade, que afeta o sexo feminino, em média, a partir dessa idade ${ }^{13}$.

Os valores de IMC das crianças e adolescentes brasileiros foram inferiores aos da OMS (P-85) em todas as idades. Embora este achado pareça ser satisfatório, pesquisas realizadas no Brasil demonstram que o país vem passando por um processo denominado "transição nutricional", o qual se caracteriza por uma inversão nos padrões de distribuição dos problemas nutricionais de uma dada população no tempo, consistindo, em geral, em uma passagem da desnutrição para o excesso de peso, nas formas de sobrepeso e obesidade ${ }^{20}$.

Comparando-se os dados do Estudo Nacional da Despesa Familiar (ENDEF), realizado em 1974 e 1975, com os dados da Pesquisa sobre Padrões de Vida (PPV), realizada em 1996 e 1997, somente nas Regiões Sudeste e Nordeste do Brasil verificou-se um aumento na prevalência de excesso de peso de 4,1 para 13,9\% em crianças e adolescentes de 6 a 18 anos $^{21}$. Estudos mais recentes realizados em diferentes cidades revelaram que a prevalência de sobrepeso variou de 8,4 a $19,0 \%$ e a obesidade, de 3,1 a $18 \%{ }^{22-25}$. Essas evidências servem de alerta para os órgãos governamentais do Brasil, que precisam planejar políticas eficazes de combate ao sobrepeso e à obesidade, pois em poucos anos as crianças e adolescentes brasileiros poderão atingir os valores de referência da OMS, os quais estão associados com agravos e riscos para a saúde ${ }^{10,22}$.

O presente estudo apresenta algumas limitações, como o delineamento transversal, que não permite o acompanhamento das variáveis ao longo do tempo em um mesmo grupo, e a seleção da amostra, que não foi formada de maneira probabilística, sendo incluídas somente as escolas que aderiram ao projeto. Entretanto, o estudo adiciona informações importantes e atualizadas sobre comparação de crianças e adolescentes brasileiros com curvas de crescimento físico empregadas internacionalmente, podendo servir como parâmetro comparativo para investigações nas diferentes regiões brasileiras.

Diante dos resultados encontrados, pode-se concluir que os valores para estatura e peso corporal das crianças e adolescentes escolares brasileiros atingem o padrão de referência da OMS e do $C D C$, respectivamente. Em relação ao IMC, os valores de referência da OMS foram superiores em todas as idades consideradas.

Sugere-se que estudos prospectivos sejam realizados verificando o crescimento físico de crianças e adolescentes do Brasil com curvas de referências internacionais a fim de poder realizar inferências mais precisas.

\section{Referências}

1. World Health Organization. Physical status: the use and interpretation of anthropometry. Report of a WHO Expert Committee. World Heatlh Organ Tech Rep Ser. 1995;854:1-452.

2. Kuczmarski RJ, Ogden CL, Grummer-Strawn LM, Flegal KM, Guo SS, Wei R, et al. CDC growth charts: United States. Adv Data. $2000 ;(314): 1-27$
3. Cacciari E, Milani S, Balsamo A, Dammacco F, De Luca F, Chiarelli $\mathrm{F}$, et al. Italian cross-sectional growth charts for height, weight and BMI (6-20 y). Eur J Clin Nutr. 2002;56:171-80.

4. El Mouzan MI, Al Herbish AS, Al Salloum AA, Foster PJ, Al Omar AA, Qurachi MM, et al. Comparison of the 2005 growth charts for Saudi children and adolescents to the 2000 CDC growth charts. Ann Saudi Med. 2008;28:334-40. Erratum in: Ann Saudi Med. 2008;28:474.

5. Mahyar A, Asefzadeh S. Comparison of weight, height and BMI of Iranian girl students with NCHS standard. NCD Malaysia. 2005;4:11-15.

6. Neyzi O, Furman A, Bundak R, Gunoz H, Darendeliler F, Bas F. Growth references for Turkish children aged 6 to 18 years. Acta Paediatr. 2006;95:1635-41. Erratum in: Acta Paediatr. 2007;96:324.

7. Sharma A, Sharma K, Mathur KP. Growth pattern and prevalence of obesity in affluent schoolchildren of Delhi. Public Health Nutr. 2007; 10:485-91.

8. Papadimitriou A. Sex differences in the secular changes in pubertal maturation. Pediatrics. 2001;108:E65.

9. de Onis M, Garza C, Onyango AW, Borghi E. Comparison of the WHO child growth standards and the CDC 2000 growth charts. J Nutr. 2007;137:144-8.

10. WHO Multicentre Growth Reference Study Group. WHO Child Growth Standards: length/height-for-age, weight-for-age, weightfor-length, weight-for- height and body mass index-for-age: methods and development. Geneva: WHO; 2006.

11. Marques RM, Marcondes E, Bequó E, Hegg R, Colli AS, Zacchi MA. Crescimento e desenvolvimento pubertário em crianças e adolescentes brasileiros II: altura e peso. São Paulo: Editora Brasileira de Ciências; 1982.

12. Brasil - Ministério da Saúde - Instituto Nacional de Alimentação e Nutrição. Perfil de crescimento da população brasileira de 0 a 25 anos: pesquisa nacional sobre saúde e nutrição. Brasília: Ministério da Saúde; 1990.

13. Malina RM, Bouchard C, Beunen G. Human growth: selected aspects of current research on well-nourished children. Ann Rev Anthropol. 1988;17:187-219.

14. Diniz IM, Lopes AS, Dummel CC, Rieger T. Crescimento físico e adiposidade corporal de escolares. Rev Bras Cineantropom Desempenho Hum. 2006;8:32-8.

15. Petroski EL, Silva RJ, Pelegrini A. Crescimento físico e estado nutricional de crianças e adolescentes da região de Cotinguiba, Sergipe. Rev Paul Pediatr. 2008;26:206-11.

16. Orellana JD, Santos RV, Coimbra Jr CE, Leite MS. Anthropometric evaluation of indigenous Brazilian children under 60 months of age using NCHS/1977 and WHO/2005 growth curves. J Pediatr (Rio J). 2009;85:117-21.

17. Gaya AC, Silva G. Manual de aplicação de medidas e testes, normas e critérios de avaliação. Porto Alegre: PROESP; 2007. http://www. proesp.ufrgs.br/institucional/index.php. Acesso: 18/04/2008.

18. Riggs BL, Khosla S, Melton LJ 3rd. Sex steroids and the construction and conservation of the adult skeleton. Endocr Rev. 2002;23:279302. Review.

19. Wells JC. Sexual dimorphism of body composition. Best Pract Res Clin Endocrinol Metab. 2007;21:415-30. Review.

20. Kac G, Velásquez-Meléndez G. A transição nutricional e a epidemiologia da obesidade na América Latina. Cad Saude Publica. 2003;19:4-5.

21. Wang Y, Monteiro C, Popkin BM. Trends of obesity and underweight in older children and adolescents in the United States, Brazil, China, and Russia. Am J Clin Nutr. 2002;75:971-7.

22. Guimarães LV, Barros MB, Martins MS, Duarte EC. Fatores associados ao sobrepeso em escolares. Rev Nutr. 2006;19:5-17.

23. Oliveira AM, Cerqueira EM, Souza JS, Oliveira AC. Sobrepeso e obesidade infantil: influência de fatores biológicos e ambientais em Feira de Santana, BA. Arq Bras Endocrinol Metab. 2003;47:144-50. 
24. Soar C, Vasconcelos FA, Assis MA, Grosseman S, Luna ME. Prevalência de sobrepeso e obesidade em escolares de uma escola pública de Florianópolis, Santa Catarina. Rev Bras Saúde Mater Infant. 2004;4:391-7.

25. Costa RF, Cintra IP, Fisberg M. Prevalência de sobrepeso e obesidade em escolares da cidade de Santos, SP. Arq Bras Endocrinol Metab. 2006;50:60-7.
Correspondência:

Edio Luiz Petroski

Núcleo de Pesquisa em Cineantropometria e Desempenho Humano

(NuCIDH) - Centros de Desportos (CDS) - Universidade Federal de

Santa Catarina (UFSC)

Campus Universitário - Trindade - Caixa Postal 476

CEP 88040-900 - Florianópolis, SC

Tel.: (48) 3721.8562, (48) 8413.4079

E-mail: petroski@cds.ufsc.br 